\title{
Beyond the volcano limitations in electrocatalysis - oxygen evolution reaction
}

\author{
Halck, Niels Bendtsen; Petrykin, Valery; Krtil, Petr; Rossmeisl, Jan
}

Published in:

Physical Chemistry Chemical Physics

Link to article, DOI:

$10.1039 / \mathrm{c} 4 \mathrm{cp} 00571 \mathrm{f}$

Publication date:

2014

Document Version

Publisher's PDF, also known as Version of record

Link back to DTU Orbit

Citation (APA):

Halck, N. B., Petrykin, V., Krtil, P., \& Rossmeisl, J. (2014). Beyond the volcano limitations in electrocatalysis oxygen evolution reaction. Physical Chemistry Chemical Physics, 16(27), 13682-13688.

https://doi.org/10.1039/c4cp00571f

\section{General rights}

Copyright and moral rights for the publications made accessible in the public portal are retained by the authors and/or other copyright owners and it is a condition of accessing publications that users recognise and abide by the legal requirements associated with these rights.

- Users may download and print one copy of any publication from the public portal for the purpose of private study or research.

- You may not further distribute the material or use it for any profit-making activity or commercial gain

- You may freely distribute the URL identifying the publication in the public portal

If you believe that this document breaches copyright please contact us providing details, and we will remove access to the work immediately and investigate your claim 
Cite this: Phys. Chem. Chem. Phys., 2014, 16, 13682

Received 7th February 2014

Accepted 11th March 2014

DOI: $10.1039 / c 4 c p 00571 f$

www.rsc.org/pccp

\section{Beyond the volcano limitations in electrocatalysis - oxygen evolution reaction $\dagger$}

\author{
Niels Bendtsen Halck, ${ }^{a}$ Valery Petrykin, ${ }^{b}$ Petr Krtil ${ }^{\mathrm{b}}$ and Jan Rossmeis ${ }^{* a}$ \\ Oxygen evolution catalysis is restricted by the interdependence of adsorption energies of the reaction \\ intermediates and the surface reactivity. The interdependence reduces the number of degrees of \\ freedom available for catalyst optimization. Here it is demonstrated that this limitation can be removed by \\ active site modification. This can be achieved on ruthenia by incorporation of $\mathrm{Ni}$ or Co into the surface, \\ which activates a proton donor-acceptor functionality on the conventionally inactive bridge surface sites. \\ This enhances the actual measured oxygen evolution activity of the catalyst significantly compared to \\ conventional ruthenia.
}

\section{Introduction}

Electrocatalytic energy conversion and storage have gained in importance recently mainly in connection with the growing role of renewable energy sources. ${ }^{1}$ Fundamentally, the underlying electrocatalytic reactions are redox processes of multi-electron nature and can be perceived as a sequence of single electron charge transfer steps. These processes are also - as a rule kinetically hindered and require a substantial energetic driving force to proceed at technologically acceptable rates.

Regardless of the nature of the electrocatalytic process, it has to follow through surface confined reaction intermediates. This means that the driving force can be minimized and the catalyst activity thereby optimized if the binding of the reaction intermediates is matched. The ideal catalyst is showing appreciable activity at virtually zero driving force. Such an "ideal electrocatalyst" needs to have equidistant distribution of the free energy in each individual charge transfer step of the whole reaction sequence. Rational catalyst design, therefore, can be viewed as an attempt to fine-tune the energetics of the charge transfer reactions to achieve the equal distribution of the free energy in all steps of the reaction sequence. ${ }^{2}$ This is equivalent to optimization of the relative strength of the intermediate(s) bonding to the catalyst surface, which can be theoretically assessed using density functional theory (DFT). ${ }^{3}$ Consequently DFT can be

\footnotetext{
${ }^{a}$ Center for Atomic-Scale Materials Design, Department of Physics, Technical University of Denmark, Building 307, $2800 \mathrm{Kgs}$. Lyngby, Denmark. E-mail: jross@fysik.dtu.dk

${ }^{b}$ Department of Electrocatalysis, J. Heyrovský Institute of Physical Chemistry, Academy of Sciences of the Czech Republic, Dolejškova 3, 18223 Prague, Czech Republic. E-mail: Petr.Krtil@jh-inst.cas.cz

$\dagger$ Electronic supplementary information (ESI) available: Experimental procedure, active site model formulation, catalytic characterization and a theoretical model of the oxygen evolution reaction. See DOI: 10.1039/c4cp00571f
}

used to estimate the driving forces needed in each individual charge transfer step. This represents the thermodynamic limit of the overall reaction kinetics. The catalyst design is, therefore, reduced to finding a material featuring optimal binding of all intermediates and consequently an optimal activity.

The real catalyst's design is, however, hindered by the interdependence of two or more reaction steps. The binding of the intermediates tends to show the same linear scaling with the catalyst's reactivity, which reduces the number of degrees of freedom (tuneable parameters) available for the catalyst's optimization. The reactivity which is the only tuneable parameter can therefore be used as an activity descriptor. Because only one tuneable parameter is available one can doubt the possibility to design catalysts approaching the thermodynamic limit.

This conceptual restriction, often described as the universality of the scaling relationship, has been verified for various electrocatalytic processes including oxygen evolution, ${ }^{4}$ oxygen reduction, ${ }^{5}$ and methanol oxidation ${ }^{6}$ as well as for various classes of the electrocatalytic materials including metals, ${ }^{7}$ oxides (sulfides, nitrides), ${ }^{8}$ and molecular catalysts. ${ }^{9}$ Therefore it seems to be an inherent limit of the rational design of electrocatalysts for the multiple electron redox processes. Breaking the scaling relationship(s) allowing for independent binding energy optimization of the reaction intermediates represents in this respect a major challenge for both theoretical and synthetic chemistry. It also represents the only way for qualitative improvement of the catalytic performance beyond the state of the art. The most intuitive approach to break the scaling relationships is to modify the active site by changing it from a surface catalyst to a three dimensional active site. ${ }^{4}$ However, so far none of the suggestions has been successfully realized.

The oxygen evolution reaction (OER) serves as a suitable model system. It combines sufficient simplicity with practical importance as OER represents the limiting process in the 
generation of hydrogen in electrochemical or photo-electrochemical water splitting. ${ }^{10}$

The oxygen evolution process is a four electron oxidation process driven by a transfer of $4.92 \mathrm{eV}$ per oxygen molecule, i.e., $1.23 \mathrm{eV}$ per electron. Experiments identify the second or third electron transfer as the rate limiting step regardless of the nature of the electrode material. ${ }^{11}$ The theoretical analysis of the problem concurs with the experimental assessment. The free energy required for the second charge transfer step is identified as an universal single descriptor of the oxygen evolution process. ${ }^{4}$ The definition of the descriptor reflects the fact that the intermediates obtained in the first and third charge transfer step show the same scaling with the surface reactivity.

Regardless of the catalyst, the free energies of the ${ }^{*} \mathrm{OH}$ and ${ }^{*} \mathrm{OOH}$ intermediates show a constant difference of approximately $3.2 \mathrm{eV} .^{4,12}$ This constant difference is $c a .0 .8 \mathrm{eV}$ higher than the desired $2.46 \mathrm{eV}$ of an ideal catalyst with equidistant free energy steps. This defines the smallest theoretically conceivable over-potential needed to drive the oxygen evolution to approximately $0.4 \mathrm{~V}$. Although the theoretical description in principle allows for catalyst design and optimization, the $0.4 \mathrm{~V}$ penalty represents a limitation applicable to all the catalysts considered so far. ${ }^{4,13,14}$ It needs to be stressed that the studies reported so far on the rational design (i.e. a combination of the theoretical prediction and targeted synthesis) to optimize the electrocatalytic activity in oxygen evolution remain within this paradigm and utilize combinatorial screening to optimize a single descriptor of the surface reactivity. The resulting materials - although offering a variability of the catalysts' electronic structure - cannot break the limitation put forward by the scaling relationship described above and their activities are summarized in the volcano curves.

Restricting the considerations to rutile type oxide catalysts, which represent industrial benchmark materials for OER, one may confine the actual activity to the so-called coordination unsaturated sites (cus) present on the surface. ${ }^{15}$ The cus sites can be identified with surface metal cations which form $(n-1)$ bonds with oxygen (where $n$ is the number of oxygen bonds formed by the given cation in the bulk). Only cus sites allow for formation of reactive "atop" positions essential for the formation of strongly adsorbed intermediates. ${ }^{15}$ of the rutile oxides ruthenia $\left(\mathrm{RuO}_{2}\right)$ is known to be particularly active in the oxygen evolution as the theoretical analysis also confirms which places this oxide close to the top of the volcano. It was reported that even in the case of ruthenia based catalysts the activity is improved by a controlled incorporation of the heterovalent cations. ${ }^{16}$ It may be envisaged that the cus site architecture may be artificially modified by the incorporation of heterovalent cations. This communication elaborates the possible effects of the local structure modifications on the resulting oxygen evolution activity and presents a general approach capable of breaking the universal scaling relationship of the OER. The general nature of this approach is demonstrated by a DFT based theoretical analysis of the OER activity of modified ruthenia catalysts combined with their experimental behaviour.

\section{Methods}

$\mathrm{Ni}$ and Co incorporated nanocrystalline ruthenia catalysts were prepared by co-precipitation of alcohol based solution of ruthenium(III) nitrosyl nitrate with a stoichiometric amount of $\mathrm{Ni}\left(\mathrm{NO}_{3}\right)_{2}$ or $\mathrm{Co}\left(\mathrm{NO}_{3}\right)_{2}$ by tetramethylammonium hydroxide. The precipitate was aged in a PTFE lined autoclave at $120{ }^{\circ} \mathrm{C}$ for 24 hours. The resulting precursor was filtered, dried and annealed at $400^{\circ}$ for 3 hours to obtain crystalline catalysts. Details of the synthesis and characterization can be found in ref. 17 and 18. The reference samples of $\mathrm{IrO}_{2}$ and $\mathrm{MnO}_{2}$ were prepared by hydrothermal synthesis from iridium(III) acetylacetonate (Alfa Aesar) and potassium permanganate (Aldrich), respectively. The electrodes for electrochemical experiments were prepared from synthesized materials by sedimentation of nanocrystalline powder from a water based suspension $\left(5 \mathrm{~g} \mathrm{~L}^{-1}\right)$ on Ti mesh (open area $20 \%$, Goodfellow) to obtain the surface coverage of about $1-2 \mathrm{mg} \mathrm{cm}^{-2}$ of active oxide. The deposited layers were stabilized by annealing the electrodes for $20 \mathrm{~min}$ at $400{ }^{\circ} \mathrm{C}$ in air. The electrocatalytic activity of the prepared materials with respect to oxygen evolution was studied in potentiostatic experiments in a $0.1 \mathrm{M} \mathrm{HClO}_{4}$ solution. All experiments were performed in a home-made Kel-F single compartment three-electrode cell controlled by a PAR 263A potentiostat. Pt and saturated calomel electrodes (SCE) were used as auxiliary and reference electrodes, respectively. All potentials shown in the text are recalculated and quoted with respect to $\mathrm{RHE}$.

The model structures used in DFT calculations were based on the local structure as obtained by the refinement of extended X-ray absorption fine structure (EXAFS) functions processed from the X-ray absorption spectra (XAS) measured on $\mathrm{Ru}, \mathrm{Ni}$ and Co $\mathrm{K}$ absorption edges. Details of these experiments are given in ESI. $\dagger$

The DFT binding energies are calculated using a software program in which the valence electronic states are described by a plane wave basis and the core-electron interactions with Vanderbilt ultrasoft pseudopotentials. ${ }^{19}$ For all surfaces the exchange-correlation functional Revised Perdew-Burke-Ernzerhof (RPBE) was used. ${ }^{20}$ The planewave basis used a cutoff of $350 \mathrm{eV}$ for the kinetic energy and a $500 \mathrm{eV}$ cutoff for the density. A $4 \times$ $4 \times 1$ Monkhorst-Pack grid was used to sample the Brillouin zone of the system. The conventional rutile ruthenia $\{110\}$ surface is modelled using a $1 \times 2$ supercell with 4 atomic trilayers as described in the literature. ${ }^{4}$ The Ni modified $\{110\}$ surface is modelled using a larger $1 \times 3$ supercell with $1 \mathrm{Ni}$ in the bridge row and $1 \mathrm{Ni}$ in the cus row as shown in Fig. 1 together with other possible local arrangements. The calculations are spin polarized.

\section{Results and discussion}

The $\mathrm{Ni}^{17}$ and $\mathrm{Co}^{18}$ incorporated ruthenia conforms apparently to a single phase rutile structure featuring an uneven distribution of the structure incorporated cation. EXAFS based structural studies prove that the $\mathrm{Ni}$ and Co cations show a strong tendency to form clusters coordinated along the (111) direction 

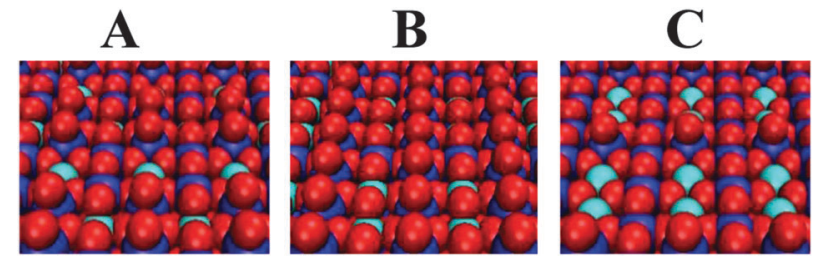

Fig. 1 Three possible schematic representations of the modified oxygen evolution active site in the Ni modified ruthenia with different placements of $\mathrm{Ni}$ on the rutile ruthenia $\{110\}$ surface: (A) one bridge and one cus $\mathrm{Ni}$, (B) two bridge $\mathrm{Ni}$ and (C) two cus $\mathrm{Ni}$. The structures were based on EXAFS refinement. ${ }^{4}$ Representation $A$ was used for the DFT calculations. Color coding of the atoms: $\mathrm{Ru}$ - blue, $\mathrm{Ni}$ - green, and $\mathrm{O}$ - red

of the rutile structure rather than distribute homogeneously in the ruthenium rich oxide framework. Despite the apparently intact translational order of the $\mathrm{Ni}$ and Co modified oxides, the cation introduction alters the local structure of the catalyst in the way shown in Fig. S1 (ESI $\dagger$ ). Regardless of the nature of the incorporated cation, the mechanism compensating for the lower charge of the $\mathrm{Ni}$ and Co cations suppresses the clustering of cations along the (001) direction. Assuming a surface structure conforming to this constraint one can construct three principal arrangements shown in Fig. 1. These may feature isolated heteroatoms in either cus or bridge position (Fig. 1A) separated by cationic sites occupied by $\mathrm{Ru}$ atoms. Alternatively one may assume the presence of short chains of the heteroatoms (2-3) stacking along the (001) direction in either bridge or cus position forming an isolated island in the surface (Fig. $1 \mathrm{~B}$ and C). ${ }^{17}$

The functionality of these principal arrangements is visualized in the DFT calculations assuming the smallest unit cell featuring all needed local arrangements (see Fig. 1A). The overall energetics of the oxygen evolution process on the Ni modified ruthenia (see Fig. 2) shows a significant deviation from that of conventional ruthenia. The potential controlling step that requires the highest driving force has changed from the third electron removal for conventional ruthenia to the second electron removal for the Ni modified ruthenia and the first electron removal for the Co modified ruthenia. The biggest free energy step amounts to $1.49 \mathrm{eV}$ and $1.33 \mathrm{eV}$ for the $\mathrm{Ni}$ and $\mathrm{Co}$ modified ruthenia, respectively, (see Fig. 2) as compared to $1.65 \mathrm{eV}$ for conventional ruthenia. This allows us to estimate the thermodynamic limit of the overpotential of the whole process to $\sim 0.3 \mathrm{~V}$ for Ni modified ruthenia and $\sim 0.1 \mathrm{~V}$ for Co modified ruthenia. These overpotentials are significantly lower than the minimum overpotential of $0.4 \mathrm{~V}$ predicted previously ${ }^{4}$ which is mainly due to lowering of the energy of the $\mathrm{S}_{3}$ state compared to conventional ruthenia as shown in Fig. 3 . In the experiments (see Fig. 4) cation modified ruthenia materials show a greater activity compared with conventional ruthenia. ${ }^{21}$

The results of the DFT modelling rationalize the effect of the introduction of $\mathrm{Ni}$ or Co on the ruthenia surface. The presence of $\mathrm{Ni}$ or $\mathrm{Co}$ at the cus positions has only negligible effect on the binding properties of the predominantly Ru composed surface. The binding energy of oxygen on the catalytically active Ru cus site is 2.75 and $2.59 \mathrm{eV}$ for $\mathrm{Ni}$ and $\mathrm{Co}$ in the bridge site, respectively, while for conventional ruthenia the binding of $\mathrm{O}$ in the same position is $2.73 \mathrm{eV}$ (see Table S1, ESI $\dagger$ and Fig. 3).
In this way the presence of $\mathrm{Ni}$ or $\mathrm{Co}$ in the cus site cannot be related to the observed increase in the oxygen evolution activity of $\mathrm{Ni}$ or $\mathrm{Co}$ modified ruthenia. The available bridge positions generally deemed non-participating in the oxygen evolution process get activated by the presence of $\mathrm{Ni}$ or $\mathrm{Co}$, which allows for simultaneous electronproton transfer at the potential close to the standard potential of the oxygen evolution reaction. The activation of the bridge site as a proton donor-acceptor effectively introduces a second tuneable parameter of the oxygen evolution process as the bridging $\mathrm{O}$ adsorbs hydrogen from the ${ }^{*} \mathrm{OH}$ in $\mathrm{S}_{1}$ and ${ }^{*} \mathrm{OOH}$ species in $\mathrm{S}_{3}$ (Fig. 2) which lowers the energies of these states compared to conventional ruthenia. The reactivity of the surface cus sites and the bridge site proton donor-acceptor potential are still weakly correlated via a hydrogen bond, which affects the oxidation potential of the bridge site if oxygen is present on the adjacent cus site. For Ni and Co modified ruthenia the potential for removing the proton from the bridging oxygen with oxygen present on the ruthenium cus site is $1.33 \mathrm{~V}$ and $1.49 \mathrm{~V}$, respectively, and without oxygen on the cus site the potential for removing the proton is 1.23 and $1.33 \mathrm{~V}$ respectively (see Table S1 in ESI $\dagger$ ). An improvement in the oxygen evolution related catalytic activity in hematite with $\mathrm{Ni}$ and Co doping has also been reported but the observed effect is rather moderate which is likely due to the semiconducting nature of hematite. ${ }^{22}$

The DFT calculations show that the $\mathrm{Ni}$ and Co modified ruthenia still do? lag behind the performance of an ideal catalyst. It is essential to stress, however, that the activation of the bridge sites removes the problem of the same free energy scaling of different intermediates providing the necessary degree of freedom to approach a global optimum via a new reaction pathway. Note that an adjustment of the donor-acceptor levels of the introduced cation is prerequisite for the oxygen evolution enhancement. Fig. 5 shows that the apex of the "volcano curve" based on the scaling relationship appears at $1.6 \mathrm{eV}$, i.e. at somewhat higher descriptor value than that of the ideal catalysts $(1.23 \mathrm{eV})$. The theoretical activity predicted for $\mathrm{Ni}$ and Co modified ruthenia appears significantly above the apex of the conventional "volcano curve". These catalysts apparently being to the weak binding leg of the volcano, which depicted by the dashed line in Fig. 5, despite their descriptor belonging to the strong binding region.

This situation can be rationalized keeping in mind that the proton/acceptor functionality represents an additional descriptor not reflected in Fig. 5. Fig. 5, therefore, represents a onedimensional reduction of a two dimensional volcano surface. In this two-dimensional approach the predicted catalytic activities would form the surface of a pyramid where the base is described by the reactivity of the surface cus sites and the bridge site proton donor-acceptor potential forming the $x$ and $y$ axes. In practical terms the introduction of the second parameter as seen for the oxygen evolution on Ni modified ruthenia, essentially outlines the simplest multi-dimensional approach allowing us to improve the electrocatalyst's behavior beyond the limitations of a single descriptor "volcano curve".

Although the experimental results do reflect an increase of the oxygen evolution activity upon modifying ruthenia with $\mathrm{Ni}$ or Co the observed effect (see Fig. 4) seems to be less significant than the theoretical predictions. 

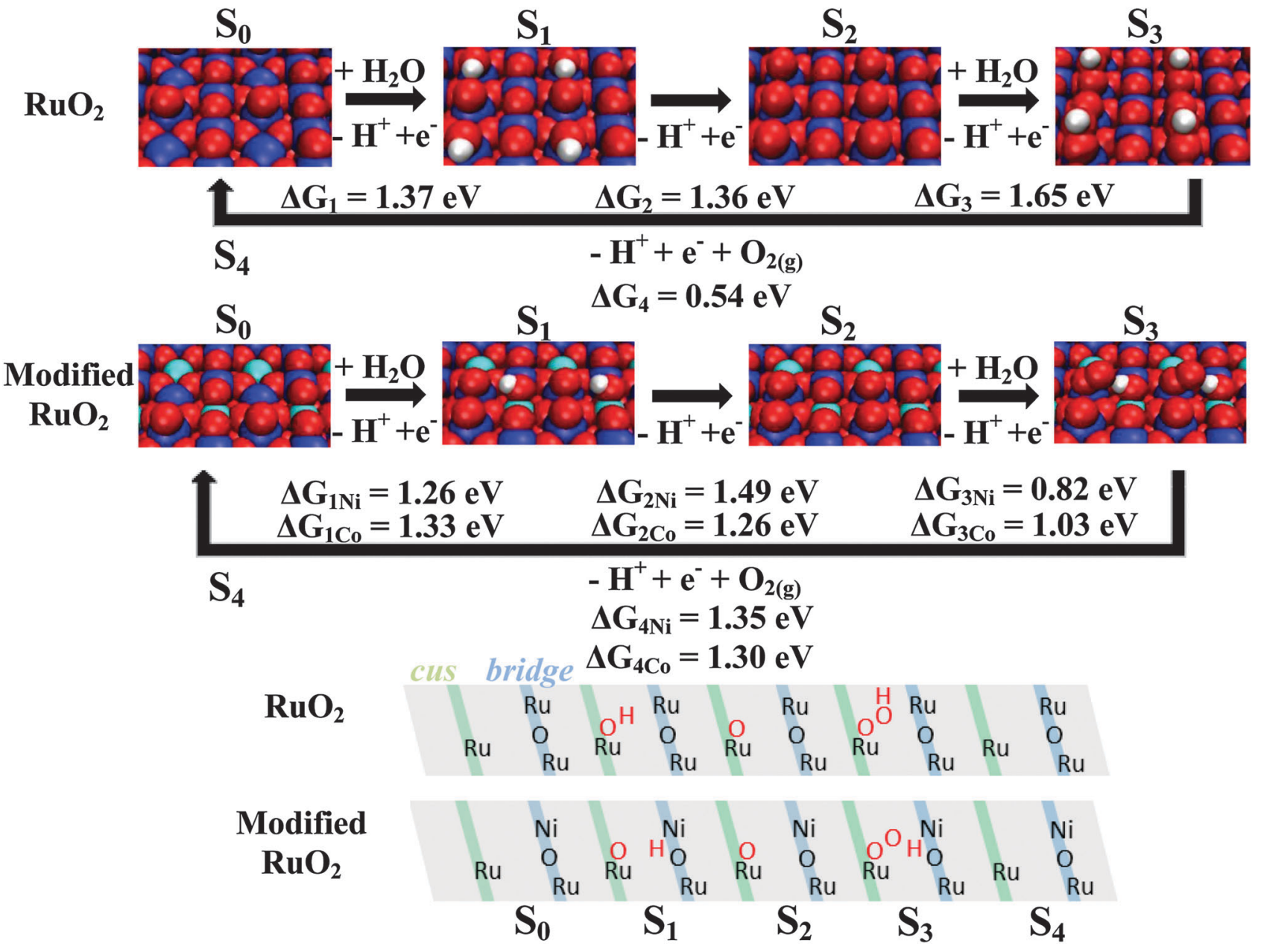

Fig. 2 Reaction mechanism of the oxygen evolution reaction on conventional rutile ruthenia and $\mathrm{Ni}$ and Co modified ruthenia on the $\{110\}$ surface. The Gibbs free energies obtained from DFT calculations for each of the reaction steps are included. For Ni and Co modified ruthenia the first and third step deviate in energy due to the activating bridging $\mathrm{O}$ atom which binds the proton. Color coding of the atoms: $\mathrm{O}-\mathrm{red}$, Ru - blue, Ni or Co - Cyan, and $\mathrm{H}$ - white. Below is a schematic figure of the role of the two binding sites for ruthenia and Ni modified ruthenia. The green row represents the cus row and the blue row represents the bridge row and the red color indicates intermediates.

This discrepancy can be qualified realizing the conceptual difference between real catalysts and their model representation in the DFT calculations. While the DFT calculations are created by periodic replications of the modified active site (see Fig. 1) the real catalysts feature only a limited number of the modified active sites diluted in the ruthenia matrix. A correct correlation in such a case can be obtained if one uses the measured current density per active site which is corrected for the contribution of the regions containing no dopant.

These current densities can be calculated using a simple formalism anticipating that the presence of each dopant atom in the bridge or cus site is proportional to the total dopant concentration. In this case the site normalized current for cobalt modified ruthenia can be written as:

$$
J\left(\mathrm{RuMe}_{\text {site }}\right)=\frac{J\left(\mathrm{RuMe}_{x}\right)-(1-x) J(\mathrm{Ru})}{x}
$$

where $x$ stands for the Ni or Co fraction, $J\left(\mathrm{RuMe}_{x}\right)$ and $J(\mathrm{Ru})$ represent experimentally measured current density for modified and conventional ruthenia, respectively, and $J\left(\mathrm{RuMe}_{\text {site }}\right)$ stands for the site normalized current density.

While the site normalized current densities of the Co modified catalysts calculated for different overall Co content according to eqn (1) are independent of concentration, the site normalized current densities of the $\mathrm{Ni}$ modified materials remain concentration dependent as shown in Fig. 6. This behavior is caused by the concentration dependence of the $\mathrm{Ni}$ local environment. In the particular case of modified ruthenia, the Co modification forms a cluster structure which is independent of concentration whereas the Ni modified ruthenia tend to form clusters protruding preferentially in the $\{110\}$ surfaces with increasing Ni content.

This clustering tendency violates the assumption expressed in eqn (1) since the probability of $\mathrm{Ni}$ entering the activated bridge position increases above the proportionality if the total Ni content $x$ exceeds 0.05 . The deviation from the proportionality may be corrected if the structure of the cluster is taken into account. EXAFS based cluster structures applicable to Co and 


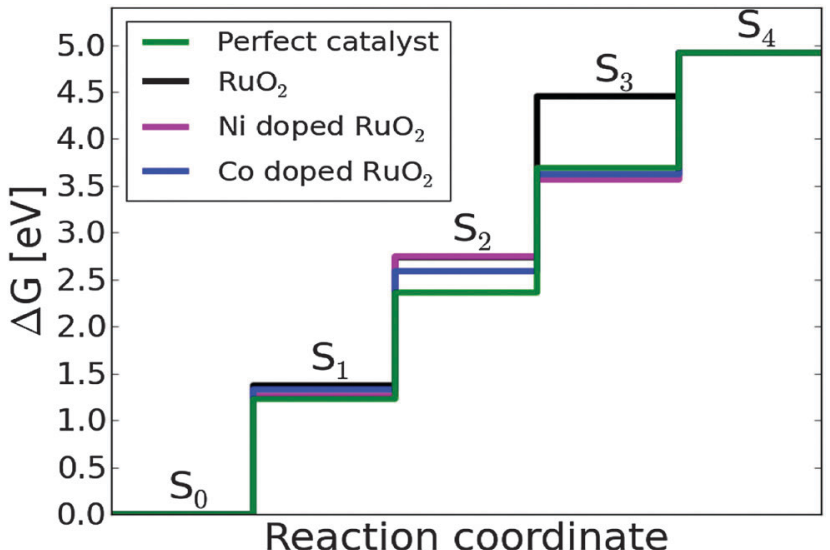

Fig. 3 Free energy diagram based on DFT calculations for conventional, $\mathrm{Ni}$ and $\mathrm{Co}$ modified ruthenia and the perfect catalyst for the four steps in the oxygen evolution reaction mechanism. The modified ruthenia catalysts have significantly stronger binding in $\mathrm{S}_{3}$ which is the potential limiting step for ruthenia.
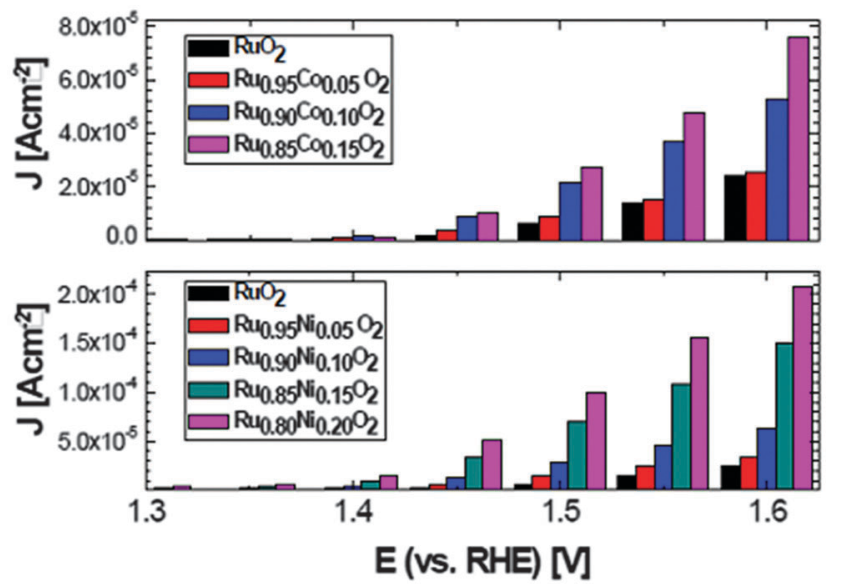

Fig. 4 The current density of oxygen evolution on $\mathrm{Ni}$ and $\mathrm{Co}$ modified ruthenia in $0.1 \mathrm{M} \mathrm{HClO}_{4}$. The data were extracted from potentiostatic experiments $40 \mathrm{~s}$ after potential application.

Ni modified structures are shown in Fig. S1 of the ESI. $\dagger$ While the structure shown in Fig. S1a (ESI $\dagger$ ) applies to all Co modified ruthenia, it only applies for the modified structures with low $\mathrm{Ni}$ content $(x=0.05)$. The structure shown in Fig. S1b (ESI $\dagger)$ is valid for Ni modified ruthenia with higher Ni content $(x=0.1)$. The cluster size can be conservatively estimated to be 3 and $5 \mathrm{Ni}$ atoms, respectively. The orientation of the clusters with respect to the $\{110\}$ surface of the nanoparticles sets a correction factor $y$ complementing eqn (1), which reflects the fraction of the cluster atoms possibly residing in the $\{110\}$ oriented surface.

$$
J\left(\mathrm{RuNi}_{\text {site }}\right)=\frac{J\left(\mathrm{RuNi}_{x}\right)-(1-x) J(\mathrm{Ru})}{x y}
$$

This correction factor is equal to $1 / 3$ and $3 / 5$ for the structures shown in Fig. S1a and b (ESI $\dagger$ ), respectively. The site normalized current densities reflecting the structure of the Co or Ni clusters are shown in Fig. 6 (blue and green symbols). The correction for

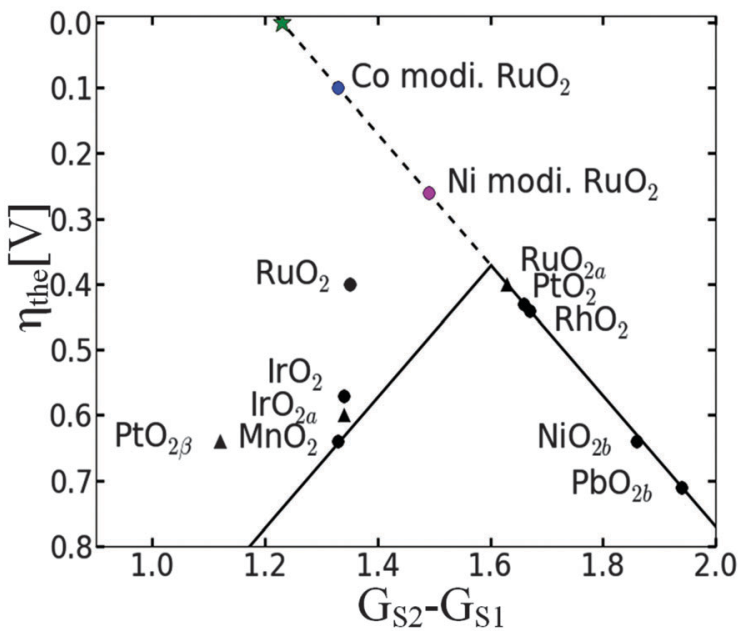

Fig. 5 Volcano curve of the theoretical overpotential for oxygen evolution processes based on the DFT calculations described in the literature ${ }^{4}$ using the second charge transfer reaction as a descriptor. The star marks the position of an ideal catalyst, the magenta circle corresponds to $\mathrm{Ni}$ modified ruthenia and the blue circle to Co modified ruthenia.

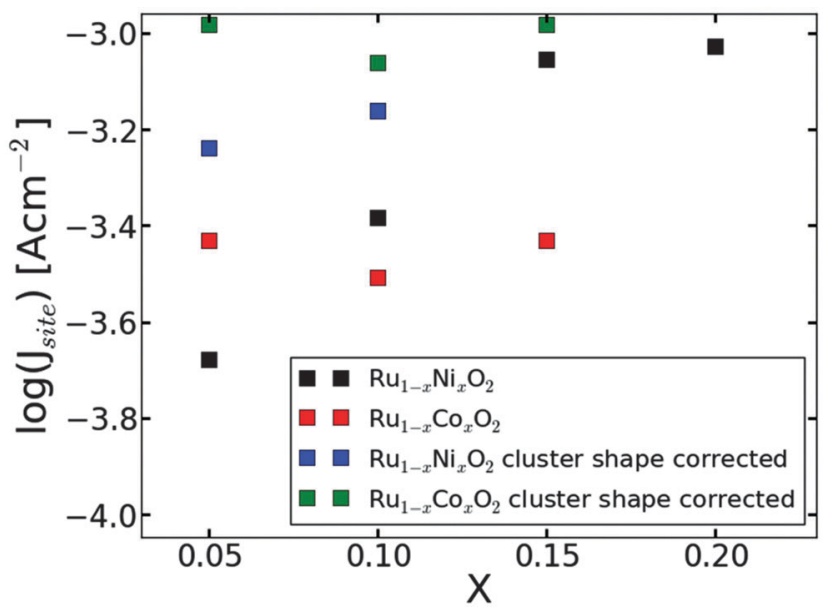

Fig. 6 Site normalized oxygen evolution activity of $\mathrm{Ni}$ and $\mathrm{Co}$ modified ruthenia $\mathrm{Ru}_{1-x} \mathrm{Ni}_{x} \mathrm{O}_{2}$ as a function of the $\mathrm{Ni}$ and $\mathrm{Co}$ content with (blue and green squares) and without cluster shape correction (red and black squares). The term log denotes the base 10 logarithm.

the size and shape suppresses the concentration dependence of Ni clusters' site normalized activity. It needs to be noted that the site normalized activity of the Co modified materials remains higher than that of the Ni counterparts, although this difference decreases with increasing concentration of the dopant. Superiority of the Co modified materials - particularly at low $x$ - generally agrees with the results of the DFT calculations.

DFT predicted thermodynamic limits to the overpotentials are often compared with the parameters used to describe the electrode kinetics - e.g. current density at the chosen electrode potential. It has to be borne in mind that DFT does not provide overpotential values that directly can be compared to experiments. Only the trends in results should be compared. This fact can be explored to compare the theoretically limiting 


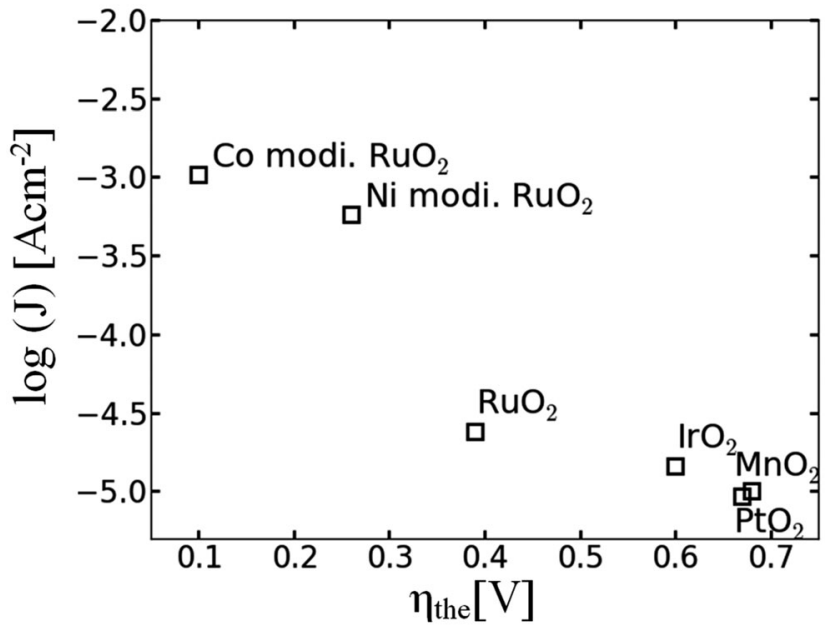

Fig. 7 The correlation between the measured current density measured at $1.6 \mathrm{~V}$ (vs. RHE) and the theoretical overpotential for different oxides. For the mixed oxides a concentration of $\mathrm{Ni}$ or $\mathrm{Co}$ of 0.05 is chosen to represent the activity per site for the cation modified ruthenia. More detailed information is available in the ESI. $\dagger$

overpotentials with the experimental current densities taken for different catalysts at the same electrode potential. Provided that electrode reaction on all compared electrode materials follows the same reaction mechanism one should reasonably assume the experimental current density to be an exponential function of the DFT predicted limiting overpotential which is shown in Fig. 7 where the dependence of the experimental current density at $1.6 \mathrm{~V}$ ( $v s$. RHE) of several known oxide electrocatalysts on the limiting activation barrier is compared. The significant increase in the site normalized oxygen evolution activity, however, also suggests rather low stability of the catalyst namely in the acid media which is indeed confirmed by the spectroscopic measurements. ${ }^{17}$

Regardless of the low stability of the Ni or Co modified ruthenia, these catalysts are the first examples of circumventing the limitations set by the scaling relationship. In this respect it needs to be accentuated that the observed phenomenon (introduction of proton acceptor-donor sites), although being an intrinsic catalyst property in this particular case, can in principle also be triggered by alternative mechanisms like, e.g. by anion ${ }^{23,24}$ or $\mathrm{CO}^{25,26}$ adsorption. This fact allows for a transfer of this approach to other electrocatalytic processes in aqueous media like, e.g. oxygen reduction ${ }^{27}$ or $\mathrm{CO}^{28}$ and $\mathrm{CO}_{2}$ reduction, ${ }^{29}$ if the electronic properties of the modified active site are fine-tuned with respect to the standard potential of the overall process. It also gives a clear indication that the rational design of the catalysts should aim at modifying the local structure of the catalytically active materials which is likely to result in metastable structures rather in stable ones which were in the center of exploration, so far.

\section{Conclusions}

Theoretical analysis of the oxygen evolution on $\mathrm{Ni}$ and $\mathrm{Co}$ modified ruthenia catalysts shows that the proton donor-acceptor functionality of the bridge site can be optimized independently of the surface reactivity at the cus sites, which results in a significant reduction of the theoretical overpotential compared to the conventional ruthenia which is also reflected in the experimental work as Ni modified ruthenia is observed to be far more active than conventional ruthenia beyond which the scaling relationship predicts.

The addition of a proton donor-acceptor functionality to the oxygen evolution reaction represents a simple multidimensional optimization of multi-electron electrocatalytic processes in aqueous media. This principle can be likely extended to other electrocatalytic processes and may represent a general concept of the rational catalyst design.

The comparison between experimental and theoretical work on modified ruthenia is complicated by the structural differences between $\mathrm{Ni}$ and Co clusters formed in the ruthenia matrix which is why the per site normalization and the cluster correction are needed to be applied before the experimental results can be compared to the per site activity obtained from DFT calculations.

\section{Acknowledgements}

The CASE initiative was funded by the Danish Ministry of Science, Technology and Innovation. Support from DCSC is gratefully acknowledged. V.P. and P.K. gratefully acknowledge the support from the Grant Agency of the Academy of Sciences of the Czech Republic under contract IAA400400906. Synchrotron experimental time has been provided by National Synchrotron Light Source (NSLS) at Brookhaven National Laboratory under project 11734.

\section{Notes and references}

1 N. S. Lewis and D. G. Nocera, Proc. Natl. Acad. Sci. U. S. A., 2006, 103, 15729.

2 J. Rossmeisl, et al., J. Electroanal. Chem., 2007, 607, 83.

3 J. K. Norskov, et al., Nat. Chem., 2009, 1, 37.

4 I. C. Man, et al., ChemCatChem, 2011, 3, 1159.

5 I. E. L. Stephens, et al., Energy Environ. Sci., 2012, 5, 6744.

6 J. Rossmeisl, et al., Energy Environ. Sci., 2012, 5, 8335.

7 F. Abild-Petersen, et al., Phys. Rev. Lett., 2007, 99, 016105.

8 E. Fernandez, et al., Angew. Chem., Int. Ed., 2008, 47, 4683.

9 F. Calle-Vallejo, et al., Phys. Chem. Chem. Phys., 2011, 13, 15639.

10 G. M. Whitesides and G. W. Crabtree, Science, 2007, 315, 796.

11 S. Trasatti, Electrochim. Acta, 2000, 45, 2377.

12 M. T. M. Koper, J. Electroanal. Chem., 2011, 660, 254.

13 J. Suntivich, et al., Science, 2011, 334, 1383.

14 M. W. Kanan and D. G. Nocera, Science, 2008, 321, 1072.

15 H. A. Hansen, et al., Phys. Chem. Chem. Phys., 2010, 12, 283.

16 V. Petrykin, K. Macounova, O. A. Shlyakhtin and P. Krtil, Angew. Chem., Int. Ed., 2010, 49, 4813.

17 V. Petrykin, et al., J. Phys. Chem. C, 2009, 113, 21657. 
18 V. Petrykin, et al., Catal. Today, 2013, 202, 63.

19 http://wiki.fysik.dtu.dk/dacapo.

20 B. Hammer, et al., Phys. Rev. B: Condens. Matter Mater. Phys., 1999, 59, 7413.

21 J. Jirkovský, et al., Electrochem. Comm., 2006, 8, 1417.

22 P. Liao, et al., J. Am. Chem. Soc., 2012, 134, 13296.

23 D. Strmcnik, et al., Nat. Chem., 2009, 1, 472.

24 R. Subbaraman, et al., Nat. Mater., 2012, 11, 550.
25 P. Rodriguez, A. A. Koverga and M. T. M. Koper, Angew. Chem., Int. Ed., 2010, 49, 1241.

26 P. Rodriguez, Y. Kwon and M. T. M. Koper, Nat. Chem., 2012, 177.

27 V. Stamenkovic, et al., Angew. Chem., Int. Ed., 2006, 45, 2897.

28 A. A. Peterson and J. K. Norskov, J. Phys. Chem. Lett., 2012, $3,251$.

29 K. J. P. Schouten, et al., Chem. Sci., 2011, 2, 1902. 